\title{
Synthesis and Characterization of Tungsten (W) Nano Particles
}

\author{
E Purushotham ${ }^{*}, 1$ and N Gopi Krishna ${ }^{2}$ \\ ${ }^{1}$ Department of Physics, SR Engineering college (Autonomous), Warangal-506 371, India. \\ ${ }^{2}$ Department of Physics, Kakatiya University, Warangal-506 009, India
}

Received 1 March 2014; Accepted 10 April 2014

\begin{abstract}
Crystallite size and lattice strains in tungsten (W) powders produced by milling have been analyzed by X-ray powder diffraction. The lattice strain $(\varepsilon)$ and Debye-Waller factor $(B)$ are determined from the half-widths and integrated intensities of the Bragg reflections. In W the Debye-Waller factor is found to increase with the lattice strain. From the correlation between the strain and effective Debye-Waller factor, the Debye-Waller factors for zero strain have been estimated for W. In the present work, Hall-Williamson method for particle size/crystallite size estimation of high energy milled W nano powder. The variation of energy of vacancy formation as a function of lattice strain has been studied.
\end{abstract}

Keywords: X-ray diffraction, lattice strain, crystallite size, Debye-Waller factor, vacancy formation energy

\section{Introduction}

Recently, chemists, physicists and materials scientists have shown great interest in the development of new methods for the synthesis of nanomaterials. Thease materials are important for further miniaturization of electric devices, nanofluids [1] to enhance efficiency of heat engines, as chemical catalysts [2,3] and lasing materials [4]. Physical and chemical properties of thease materials are highly size dependent. Therefore, it is important to develop novel techniques for the synthesis of nanomaterials. TEM is one of the powerful techniques for crystallite size measurement, it has certain limitations. Since TEM images represent only a local region, many samples and images are required to provide an average information for the entire sample. Not only this, the TEM sample preparation method is an involved and time consuming one. The XRD technique is free from these limitations. X-ray diffraction is, on the other hand, a simple and easier approach for determination of crystallite size of powder samples.

The Debye-Waller factor is an important lattice dynamical property. There is considerable X-ray work on the Debye-Waller factors of W [5-7]. But it is interesting to study the effect of lattice strains on the Debye-Waller factors of this metal. Inagaki et al $[8,9]$ showed that in several nonmetallic powders, the strains produced during grinding have a significant effect on the Debye-Waller factors measured from X-ray diffraction intensities. Sirdeshmukh et al [10] observed the effect of lattice strains on the Debye-Waller factors in semiconductor powder materials. Gopi Krishna and Sirdeshmukh [11] studied the effect of lattice strains on the Debye-Waller factor of ytterbium metal. In the present

* E-mail address: psm45456@gmail.com

ISSN: 1791-2377 (C) 2014 Kavala Institute of Technology. All rights reserved. investigation the results of a systematic study of the effect of lattice strains on the Debye-Waller factors and crystallite size of face centred cubic $\mathrm{W}$ metal is reported. These results are being reported for the first time.

\section{Experimental}

The powder samples were obtained by gently filing highly pure W metal ingots with a jeweller's file. A part of this powder was used to prepare the initial sample. The remaining powder was subjected to milling in a ball mill for $5,10,15,20,25,30,35$ and 40 hours to produce strains. Xray diffractograms were recorded with the initial sample and with samples prepared after each spell of milling. The diffractograms were obtained with a Philips CWU 3710 Xray powder diffractometer in the $2 \theta$ range $20-120^{\circ}$ using filtered $\mathrm{CuK}_{\mathrm{a}}$ at a goniometer speed of 0.5 per minute and a chart speed of $20 \mathrm{~mm} / \mathrm{min}$. All measurements were made at room temperature. The observed integrated intensities have been corrected for thermal diffuse scattering using the method of Chipman and Paskin [12].

\section{Analysis of Data}

For the relative intensity method, the expression for the observed intensities $\mathrm{I}_{0}$ is given by

$$
\mathrm{I}_{0}=\mathrm{CL}_{\mathrm{p}} \mathrm{JF}_{\mathrm{T}}^{2}
$$

where $\mathrm{L}_{p}$ is the Lorentz-polarization factor, $\mathrm{J}$, the multiplicity factor, $\mathrm{F}_{\mathrm{T}}$ the structure factor and $\mathrm{C}$ is a constant. For a flat powder specimen, the absorption correction is independent of the angle $\theta$, Klug and 
Alexander[13] and, hence, is lumped with the constant. The structure factor $\mathrm{F}_{\mathrm{T}}$ in terms of the structure factor $\mathrm{F}$ for the static lattice is given by

$\mathrm{F}_{\mathrm{T}}=\mathrm{Fe}^{-\mathrm{M}}$

We may also write Eq. (1) as

$$
\mathrm{I}_{0}=I_{c} e^{-2 B\left(\frac{\sin \theta}{\lambda}\right)^{2}}
$$

where $I_{c}$ is the intensity corresponding to the static lattice and is given by

$$
\mathrm{I}_{\mathrm{c}}=\mathrm{L}_{\mathrm{p}} \mathrm{JF}^{2}
$$

For crystals with fec structure, the structure factor $\mathrm{F}$ is given by

$F=4 f$

Cromer's [14] recommendation who suggested that the atomic scattering factors given in the International Tables for X-ray Crystallography [15] which are computed from the Hartree-Fock wave functions are the best for the lighter atoms or ions in the periodic table up to $\mathrm{Rb}^{+1}$. For the heavier atoms or ions Cromer recommends the use of the Dirac-slater relativistic scattering factors given by Cromer and Waber [14].

From Eq. (3) it can be seen that $\log \left(\mathrm{I}_{0} / \mathrm{I}_{\mathrm{c}}\right)$ is linearly related to $(\sin \theta / \lambda)^{2}$. By a least square treatment of data, $B$ was determined. From the Debye-Waller theory

$\mathrm{B}=\left(\frac{8 \pi^{2}}{3}\right)<\mathrm{u}^{2}>$

for a cubic crystal, where $<\mathrm{u}^{2}>$ is the mean-square amplitude of vibration. Further, B, may also be expressed as

$\mathrm{B}=\left(\frac{6 h^{2}}{m k_{B} T}\right) \mathrm{W}(\mathrm{x})$

where $\mathrm{m}$ is the mass, $\mathrm{T}$ the absolute temperature and $\mathrm{h}$ and $\mathrm{k}_{\mathrm{B}}$ are the Planck and the Boltzmann constants respectively. The function $\mathrm{W}(\mathrm{x})$ is given by

$\mathrm{W}(\mathrm{x})=\left[\frac{\phi(x)}{x^{2}}+\frac{1}{4 x}\right]$

where $\varnothing(x)$ is the Debye function and $x=\theta_{M} / T, \theta_{M}$ being the Debye temperature. Benson and Gill [17] have tabulated values of $\mathrm{W}(\mathrm{x})$ for a wide range of $\mathrm{x}$ for small increments, from which $\theta_{M}$ can be obtained from the value of $B$.

\section{Lattice strain and Particle size determination}

When the size of the individual crystals is less than about $100 \mathrm{~nm}$ the term "particle size" is usually used. When the crystallites of a material are smaller than $100 \mathrm{~nm}$, they have too small a number of parallel diffraction planes and so they produce broadened diffraction peaks instead of a sharp peak. Lattice strain present in the sample is another cause of broadening of Bragg diffraction peaks. In addition to this, there are instrumental factors such as unresolved $a_{1}$ and $a_{2}$ peaks, imperfect focusing which lead to the (Wine broadening. There are various methods in practice to estimate the particle size. X-ray diffraction is a simpler and easier approach for the determination of precise particle size and the lattice strain in powder samples. The principle involved in the Xray diffraction approach is precise quantification of the broadening of the Bragg diffraction peaks. Scherrer equation, Hall-Williamson method and Warren-Averbach method are some of the techniques based on this principle. Of the above methods, Scherrer equation method for the estimation of particle size does not take into account the broadening due to lattice strain present in the sample. As such in the present investigation, the lattice strains have been estimated using Hall-Williamson method. Recently, Bharati et al [18] have used this method to estimate the lattice strain and particle sizes of silver nano particles and composite silver nano particles. In this method the 5 infegral breadth of the diffraction peak is determined. The integral breadth is given by the integrated intensity divided by the maximum intensity. Thus, the observed peak broadening $\mathrm{B}_{\mathrm{o}}$ may be represented as

$\mathrm{B}_{\mathrm{o}}=\mathrm{B}_{\mathrm{i}}+\mathrm{B}_{\mathrm{r}}$

where $B_{0}$ is the observed peak broadening in radians, $B_{i}$ is the instrumental broadening in radians and $B_{r}$ is the broadening due to the small particle size and lattice strain. The instrumental broadening has been estimated using a pure strain-free fine sodium chloride powder sample subjected to XRD under identical conditions as those for the strained metallic powders. Eq. (9) holds good if the diffraction peaks exhibit purely Cauchy profile. However, when the diffraction peaks are partly Cauchy and partly Gaussian for profiles, the following relation between $B_{0}, B_{i}$ and $\mathrm{B}_{\mathrm{r}}$ holds good, Bharati et al [18].

$\mathrm{B}_{\mathrm{r}}=\left[\left(\mathrm{B}_{\mathrm{o}}-\mathrm{B}_{\mathrm{i}}\right)\left(\mathrm{B}_{\mathrm{o}}^{2}-\mathrm{B}_{\mathrm{i}}^{2}\right)^{1 / 2}\right]^{1 / 2}$

Now, according to Scherrer equation, the broadening due to small particle size may be expressed as

$\mathrm{B}_{\mathrm{c}}=\frac{\mathrm{k} \lambda}{\mathrm{t}} \cos \theta$

where $B_{c}$ is the broadening solely due to small crystallite size, $\mathrm{K}$ a constant whose value depends on particle shape and usually taken as unity, $\mathrm{t}$ the crystallite size in nanometers, q the Bragg angle and 1 is the wavelength of incident X-ray beam in nanometers.

Similarly, according to Wilson [19], the broadening due to lattice strain may be expressed by the relation,

$\mathrm{B}_{\mathrm{s}}=\varepsilon \operatorname{tanq}$

where $\mathrm{B}_{\mathrm{s}}$ is the peak broadening due to lattice strain and $\varepsilon$ the strain distribution within the material and $\mathrm{q}$ is the Bragg angle. Based on Eqs. (11) and (12) the total peak broadening $\mathrm{B}_{\mathrm{r}}$ may be expressed as, 


$$
\mathrm{B}_{\mathrm{r}}=\frac{\mathrm{k} \lambda}{\mathrm{tcos} \theta}+\varepsilon \tan \theta
$$

which can be written as

$$
\mathrm{B}_{\mathrm{r}} \cos \mathrm{q}=\frac{\mathrm{k} \lambda}{\mathrm{t}}+\varepsilon \sin \theta
$$

The plot of $B_{r} \operatorname{cosq} / 1$ versus sinq/l is a straight line with slope equal to $\varepsilon$ and hence the particle size ' $t$ ' can be estimated from the intercept. Typical Hall-Williamson plot between $B_{r}$ cosq/l and sinq/l is shown in Fig. (1).

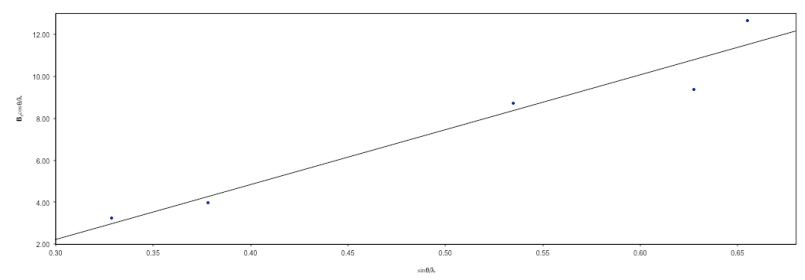

Fig. 1. Plot of $B_{r} \cos \theta / \lambda$ Vs $\sin \theta / \lambda$ for $W$ after milling for 25 hours.

The lattice strains were determined from the plot of $\mathrm{B}_{\mathrm{r}} \cos \theta / \lambda$ against $\sin \theta / \lambda$ following standard procedures [20]. The measured half-widths were corrected for instrumental broadening with reference to a pure strain-free silicon powder. The variation of particle size with milling time is within the limits of experimental errors. This shows that while the milling is enough to create strains, it affect the particle size to a measurable extent. A typical HallWilliamson plot is shown in Figure 1 for $\mathrm{Ag}$ after grinding for 25 hours.

\section{Results and discussion}

The values of the lattice strain, crystallite size, root mean square amplitude of vibrations, Debye-Waller factor and Debye temperature of $\mathrm{W}$ powders, ground for different durations, obtained in the present study are given in Table 1. As the objective of the present work is to investigate the strain dependence of Debye-Waller factors, the variation of the lattice strain $(\varepsilon)$ and Debye-Waller factor (B) for different milling times for fcc metal $\mathrm{W}$ is shown in Figure 2. Both lattice strain and Debye-Waller factor increase with milling time. This is similar to the observations of Inagaki et al [8,9], Sirdeshmuch et al [10] and Gopi Krishna and Sirdeshmukh [13]. The Debye-Waller factor increases with milling time and lattice strain in a slightly non-linear fashion and crystallite size decreases with milling time. An extrapolation of the $\mathrm{B}$ versus $\varepsilon$ curve to $\varepsilon=0$ gives the values of Debye-Waller factor $0.12 \AA^{2}$. The zero-strain values of Debye-Waller factor is less than the values for the initial samples. The zero strain Debye-Waller factors of 0.12 $\AA^{2}$ for $\mathrm{w}$ is close to the value of $0.18 \AA^{2}$ obtained by Paakkari and Scortti [5] using powder X-ray diffraction. Thus, the Debye-Waller factors of w powder samples carry an effect due to lattice strain. While comparing the DebyeWaller factors calculated from the lattice dynamical models with experimental results, Vetelino et al [21] have attributed the difference to inaccuracies in the experimental values caused by neglecting the TDS corrections. The repeated milling of the powder sample leads to lattice distortion which gives rise to microstrains in the lattice. These microstrains increase the contribution of static component of Debye-Waller factor. Thus both lattice strain and the observed Debye-Waller factor, which is the sum of static and thermal components, increase with milling time. Thus, whenever Debye-Waller factors are determined from $\mathrm{X}$-ray intensities on powder samples, it is desirable to make an estimate of the lattice strain and if the strain is large, a suitable correction is to be made as done in the present study. The Debye temperatures derived from the zero strain values of Debye-Waller factor of $\mathrm{w}$ is $386 \mathrm{~K}$. This value agree well with the values of $377 \mathrm{~K}$ [6], obtained for $\mathrm{w}$ from the powder X-ray diffraction.
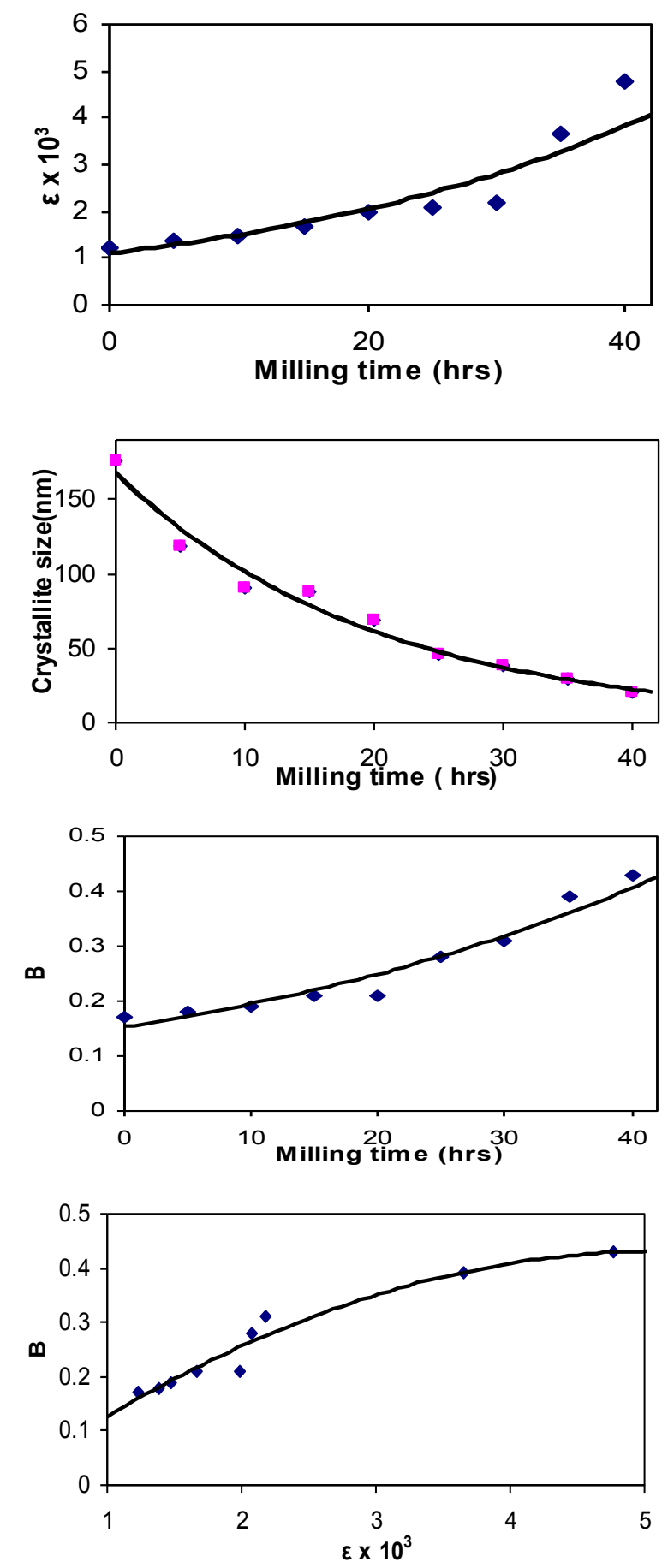

Fig. 2. Milling time $V_{S}$ lattice strain, Crystallite size and Debye-Waller factor and lattice strain $V s$ Debye-Waller factor curves for $\mathrm{W}$. 
Glyde [22] derived the following relation between the energy of vacancy formation $\left(E_{f}\right)$ and the Debye temperature $(\theta)$ of a solid. The relation is

$$
\mathrm{E}_{\mathrm{f}}=\mathrm{A}(\mathrm{k} / \mathrm{h})^{2} \mathrm{M} \theta^{2} \mathrm{a}^{2}
$$

where a is the interatomic spacing, A a constant shown to be equal to $1.17 \times 10^{-2}$, M the molecular weight and $h$ and $k$ are the Plank's and the Boltzmann's constants, respectively. Glyde recommended the use of X-ray based values in eq. (1). The validity of eq.(1) was verified for a number of fcc, bcc and hcp metals [23]. Therefore, the X-ray Debye temperatures obtained in the present work have been used to study the variation of vacancy formation energy as a function of lattice strain in W. The values of vacancy formation energies are also included in Table 1.
Table 1. Values of lattice strain $(\varepsilon)$, crystallite size $(t)$, Debye-Waller factor (B), root mean square amplitudes of vibration $<\mathrm{u}>$, Debye temperature $\left(\theta_{\mathrm{M}}\right)$ and energy of vacancy formation $\left(\mathrm{E}_{\mathrm{f}}\right)$ of strained tungsten nano powders.

Metal Milling time $\varepsilon \times 10^{3} \mathrm{t}(\mathrm{nm}) \quad<\mathrm{u}>(\AA) \quad B\left(\AA^{2}\right) \quad \theta_{\mathrm{M}}(\mathrm{K}) \quad \mathbf{E}_{\mathrm{f}}(\mathrm{eV})$

\begin{tabular}{cccccccc}
\hline $\mathrm{W}$ & 0 & 1.23 & 176 & $0.0804(3)$ & $0.17(2)$ & $336(3)$ & 7.17 \\
5 & 1.38 & 118 & $0.0830(2)$ & $0.18(1)$ & $325(5)$ & 6.71 \\
& 10 & 1.47 & 91 & $0.0850(3)$ & $0.19(1)$ & $318(3)$ & 6.42 \\
15 & 1.67 & 88 & $0.0893(4)$ & $0.21(2)$ & $303(13)$ & 5.83 \\
20 & 1.99 & 69 & $0.0935(6)$ & $0.21(2)$ & $288(9)$ & 5.27 \\
& 25 & 2.08 & 46 & $0.1031(6)$ & $0.28(1)$ & $261(6)$ & 4.33 \\
30 & 2.18 & 38 & $0.1085(4)$ & $0.31(3)$ & $248(23)$ & 3.91 \\
35 & 3.66 & 29 & $0.1217(6)$ & $0.39(4)$ & $220(31)$ & 3.07 \\
40 & 4.77 & 21 & $0.1221(4)$ & $0.43(1)$ & $216(3)$ & 2.96 \\
\hline
\end{tabular}

\section{Conclusion}

Tungsten powder was strained by milling for 40 hours. From a study of X-ray diffractograms recorded at different stages of milling, it is observed that milling for 40 hours has systematic effect on the particle size. However, the milling produces lattice strain and also enhances the effective Debye-Waller factor. By an extrapolation of the plot between the Debye-Waller factor and the lattice strain, the zero strain Debye-Waller factors are obtained for W. The variation of energy of vacancy formation as a function of lattice strain has been studied.

\section{References}

[1] Abid, J.P., Girault, H.H. and Brevet, P.F., Chem. Commun. (2001) 829 .

[2] Darugar, Q., Qian, W. and El-Sayed, M.A., J. Phy. Chem. B110 (2006) 143.

[3] Eastman,J.A., Cnoi, S.U.S., and Li, S., Yu, W. and Thompson, L.J., Appl. Phy. Lett. 78 (2001) 718.

[4] Fujiwara, H., Yanagida, S., and Kamat, P.V., J. Phy. Chem. B103 (1999) 2589.

[5] Paakkari, T. and Suortti, P. (1967). Acta Cryst. 22,755.

[6] Walford, L.K., Carron, G.J. and Schoeffel, J.A. (1968). Mat. Res. Bull. 3, 911

[7] Paakkari, T. (1974). Acta Cryst. A30, 83..

[8] Inagaki, M. Furuhashi, H.,T Ozeki et al., J Mater Sci. 6, (1971) 1520 .

[9] M Inagaki, H Furuhashi, T Ozeki and S Naka J.Mater. Sci. 8, (1973) 312.

[10] Sirdeshmukh, D.B., Subhadra, K.G., Hussain, K.A., Gopi Krishn a, N., and Raghavendra Rao. B., Cryst.Res.Technol 28, (1993) 15.

[11] Gopi Krishna, N., and Sirdeshmukh., D.B., Indian J Pure \& Appl Phys.31, (1993) 198.

[12] Chipman, D.R., and Paskin, A., J.Appl. Phys. 30, (1959) 1938.

[13]Klug, H.P., and Alexander, L.E., (1974). X-ray Diffraction Procedures (John Wiley and Sons, U.S.A.).

[14] Cromer, D.T., and Waber, J.T., Acta Cryst. 18, (1965) 104.

[15] International Tables for X-ray Crystallography (1968) Vol. III

(Kynoch Press, Birmingham).

[16]Cromer, D.T., and Liberman, D., J. Chem. Phys. 53, (1970) 1891.

[17]Benson, G.C., and Gill, E.K., (1966) Table of Integral Functions Related to Debye-Waller factor, National Research Council of Canada, Ottawa.

[18]Bharati, R., Rehani, P.B., Joshi, Kirit N., Lad and Arun Pratap, Indian Journal of Pure and Applied Physics, 44, (2006) 157-161.
[19] Wilson, A.J.C., (1949). X-ray Optics (Methuen, London). [20]Kaelble, E.F., Handbook of X-rays (New York Mc Graw ill) (1967) [21] Vetelino, J.F., Gaur, S.P., Mitra, S.S., Phys. Rev. B5, (1972) 2360. [22] Glyde, H.R., J.Phys and Chem Solids (G.B) 28, (1967) 2061.

[23] Micro-and Macro-Properties of Solids (Springer Series in Material Science) (2006) 